\title{
Design of Interoperable Communication Architecture for TSO-DSO Data Exchange
}

\author{
Nermin Suljanović, \\ Andrej Souvent \\ Elektroinštitut Milan Vidmar (EIMV) \\ Ljubljana, Slovenia
}

\author{
Gareth Taylor, Mohammed Radi \\ Brunel Institute of Power Systems, \\ Brunel University London, UK
}

\author{
Jérôme Cantenot, Eric Lambert, \\ Hugo Morais \\ EDF Lab Paris-Saclay \\ Palaiseau, France
}

\begin{abstract}
This paper describes a model driven methodology in order to implement an interoperable communication architecture supporting TSO-DSO information exchange. The model driven methodology goes through Smart Grid Architecture Model interoperability layers and leverage international standards. The Use Case approach is utilized for identification of information exchange requirements, which are materialized through Business Objects gap analysis against existing standardized IEC CIM (Common Information Model) profiles. Determined set of standardized Business Objects can be implemented using several communication technologies. Some of these up-to-date technologies are provided by off the shelf solutions such as ECCo SP, a secure and scalable platform provided by ENTSO-E.
\end{abstract}

Index Terms-- data exchange, Distribution System Operator (DSO), Transmission System Operator (TSO), TSO-DSO collaboration, use case methodology.

\section{INTRODUCTION}

The future energy landscape, in which a large amount of consumption will be covered by renewables connected to distribution grids while consumers will actively participate in the electricity market by using their flexibilities for their own benefits, drives transmission system operators (TSOs) to implement mechanisms ensuring closer and efficient collaboration with distribution system operators (DSOs) and vice versa [1]. The need for more collaboration is originated from the TSO and DSO roles, in the EU regulation perspective. While TSO is responsible for system balancing and security, the majority of distributed resources are connected to the distribution grid. On the other hand, DSO is responsible for the operation and maintenance of the distribution grid. DSO must provide a safe operation of a given control area and collaborate with TSO to maintain safe operation of the transmission system [2]. Furthermore, EU fosters competition and free transactions at electricity market requiring indiscriminate access to network and all necessary information. This makes TSO's role of neutral market facilitator more challenging, since activation of procured generation capacities at electricity market connected to the distribution grid shouldn't cause any violation of technical constraints in the transmission and distribution grids, such as congestion of power lines. TSOs and DSOs are encouraged to strengthen their collaboration and face with the common challenges. Such collaboration ultimately needs high-quality data timely exchanged between TSO and DSO in a structured, harmonized and standardized manner [3].

The exchange of information between TSOs and DSOs has been a common practice in Europe, however an increased need for interoperability between them requires improving current levels of data exchange [4]. Better TSO-DSO data exchange will not only enable more efficient network operation and planning of transmission and distribution networks but will also lower the barriers for the participation of emerging actors (aggregators, ESCOs...) in electricity markets, as well as facilitating new business models and opportunities (demand and generation flexibility management) [5]. While TSO can benefit from distributed energy resources in the distribution grid, coordination between TSO and DSO is necessary for safe, reliable, and cost-efficient use of flexibility-based services [6].

TSO and DSO collaboration and data exchange between them is an inevitable part of the overall smart grid paradigm, in which information and communication technologies are serving to power system and face the new challenges. Smart Grid Architecture Model (SGAM) framework is aimed to address interoperability in smart grids and aggregates interoperability categories into five abstract interoperability layers [7]. The SGAM allows partitioning of information management viewpoint at all layers into physical domains of electricity supply chain and hierarchical zones of the electrical processes' management. While domains cover all electricity supply chain, from customers to generation, zones represent "a hierarchical model which considers the concept of aggregation and functional separation in power system management" [7]. The aim of this paper is to present collaboration and information exchange between TSO and DSO in future energy landscape from the smart grid perspective. SGAM framework is used to put TSO-DSO collaboration aspects into the smart grid context.

The paper is organized as follows; the second section explores the common challenges posted to TSOs and DSOs and brings these challenges into smart grid concept utilizing SGAM. Classification of data exchanged between TSO and DSO is presented in the third section, emphasizing stakeholders needs and functionalities offered through data exchange platforms. Use-case methodology for identification of data exchanged between TSO and DSO, and principles how these data are modeled, are given in the fourth section. The 
last section proposes interoperable ICT architecture for TSODSO data exchange that merges inherited systems, such as SCADA (Supervisory Control And Data Acquisition) and ICCP (Inter-Control Center Communications Protocol), with new platforms, such as ECCo SP (ENTSO-E Communication And COnnectivity Service Platform) and Internet of Things (IoT) communication principles and protocols.

\section{TSO-DSO COLLABORATION IN THE SMART GRID CONTEXT}

Facing with the additional challenges and complexity, TSOs and DSOs will need to intensify their collaboration in a more regular and harmonized way. Their actions should be coordinated in order to avoid disturbances for all grid users. Despite the existing diversity of TSOs and DSOs throughout Europe, a set of common challenges can be identified [3]. The following three challenges posed to both TSOs and DSOs stand out as the most relevant:

- Enhanced grid observability and controllability;

- Integration of large amounts of variable renewable energies;

- Facilitation of flexibility services and consumer participation in electricity markets.

In the context of smart grids, listed technical challenges affect Domains and Zones at the functional layer of SGAM, as is shown in Fig. 1.

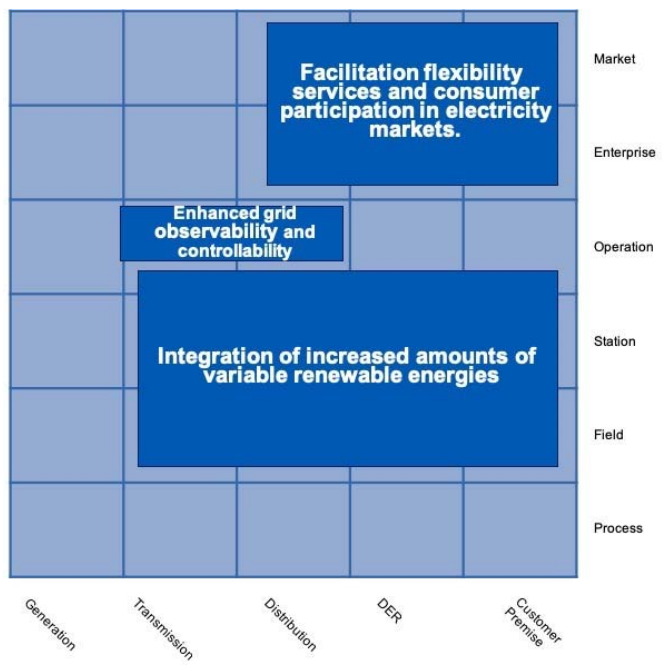

Figure 1. TSO-DSO challenges in the context of smart grid

\section{A. Enhanced grid observability and controllability}

An increased integration of distributed energy resources, in particular connected to distribution networks, changing traditional power flows, is placing new challenges to TSOs [8] and DSOs [9] in terms of limited network visibility and controllability. The transition from traditional generation to variable renewable energy sources that meet large amount of demand introduces the lack of generation controllability. For instance, TSOs are missing observability over Significant Grid Users (SGUs), such as distributed generation or demand facilities, connected to the lower voltage levels at the distribution network, with an impact on transmission networks [10]. Traditionally, DSOs have none or very limited visibility over transmission systems and TSOs' actions and realize the need for additional observability over transmission networks and connected energy resources to adequately fulfil their roles [10]. TSOs and DSOs are tending to improve the existing level of observability on each other's networks, but with a special concern on the TSO-DSO interface where actions taken by each of the system operators can affect the operations of the other system operator [9, 10].

\section{B. Integration of large amounts of variable renewable energies}

Over the past decade, the generation portfolio in Europe has changed significantly from essentially fossil fuel-based generation to a more sustainable one in which renewable energies already play an important role [11]. In particular, renewable energies of a variable nature, such as wind or solar power, have been connected at an accelerated pace in the European electricity system. TSOs and DSOs have been key enablers in this transition.

So far, TSOs and DSOs have been able to deal with challenges posed by this type of generation that is being connected at lower voltage levels in a much more distributed manner. However, one less positive aspect about distributed renewable energies is that they are being connected to the grid, but not integrated into grid operations [12]. This is an unsustainable arrangement if much higher amounts of variable renewable energies are to be connected in future European electricity system. In supporting the connection of variable renewable energies, traditional generation continues to play an important role in Europe.

\section{Facilitation of flexibility services and consumer participation in electricity markets}

The proliferation of distributed generation, together with a new paradigm shift in which some electricity consumers are willing to generate and manage their own electricity, opens up a set of new opportunities to manage the electricity system in a much more flexible way. Leveraging the existing value at the consumer side, namely by facilitating their non-discriminatory access to electricity markets, is a high priority at EU level [4, 13]. Nevertheless, the existing potential at distributed generation and consumer side to provide flexibility services to the electricity system can be most exploited in Europe.

Acting as neutral actors, responsible for ensuring the secure and reliable operation of the electricity infrastructure, TSOs and DSOs will be crucial in facilitating the participation of distributed flexibility resources in the different electricity markets (energy, system services, balancing ...) [7, 9]. They will also likely need to procure available flexibility services to operate their networks efficiently and cope with an ageing infrastructure, thus minimizing the costly measure of grid reinforcements [8-10]. There are available different coordination schemes between TSO and DSO that determine roles and responsibilities of systems operators in procurement and usage of flexibility services [6]. 


\section{CLASIFICATION OF DATA EXCHANGED BETWEEN TSO AND DSO}

Smart Grids concept relies significantly on the interaction between the energy sector and ICT infrastructure in order to deliver a reliable data access for the all the stakeholders in the electricity field. The deployment of the innovative services within the electricity network and market raises complex issue and challenges that need to empower more interactions between the stakeholders based on effective and efficient ways in handling the data access. The main data categories are described in this section and presented at the information layer of SGAM (Fig. 2).

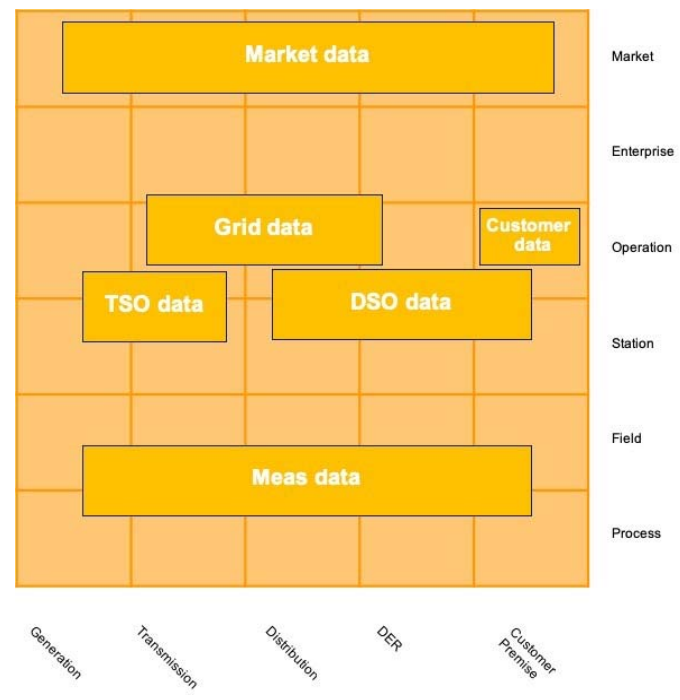

Figure 2. Data types in the SGAM context

This type of data includes all data related to the state of the grid, such as measurements, configurations and grid models. Grid data include all technical data (e.g. voltage, current, power, frequency) obtained from various measurement devices and sensors (including smart meters) and structural data (general information from the network and its relevant users, necessary for the models used to perform operational security analysis). System operators are owners of this data, which are classified as real-time, planning and historic. The basic classes of the grid data are $[4,13]$ :

- Real-time data. This class of data is related to various measurements of voltages, currents, active and reactive power, consumption, frequency and grid configuration (state of circuit breakers).

- Schedule data. Information from the grid and grid users for day ahead and intra-day, necessary for operational security analysis during this timeframe of operational planning.

- Historic measurements. Historic grid measurements that incorporate quantities that are also measured in real time (e.g. voltage, frequency, power, consumption) and have a value for off-line analysis and planning.
- Planed grid configuration. It is related for a shortterm and long-term grid planning (e.g. the day ahead operation of grids).

- Known outages. This data type refers to known outages that impacts grid configuration, generation and consumption.

- Planed grids expansions (grid reinforcement). Documents describing plans for long-time grid expansion, e.g. installations of new substations and switchgears.

- Emission data. This data is related to the environmental impact of traditional electricity generation (e.g. The Emissions Database for Global Atmospheric Research (EDGAR) by EU's Joint Research Centre).

Grid data is dominantly generated by system and grid operators, while data users are system and grid operators, aggregators, generation operators, flexibility providers, consumers and producers. TSO and DSO exchange grid data which are related to the power system operation, harmonisation of TSO and DSO actions, exchange grid models, coordinate outage plans and maintenance activities.

1) Transmission Grid Data

Grid data in the transmission grid are classified as structural data and real-time data. Structural data provide information about the grid topology and provide input for different models necessary for system estimation and dynamic stability assessment. Real-time data are related to measurement or estimated voltage, actual substation topology, regulating position of transformers, active and reactive power measurements in transformer bay, status of circuit-breakers and switching devices etc.

\section{2) Distributtion Grid Data}

DMS (Distribution Management System) utilizes SCADA measurement data applications that provide greater visibility of distribution grid and better management of DERs. Smart metering data are the basis for the short-term and long-term load forecasting together with metrological and satellite information.

\section{B. Electricity Market Data}

Neutral and efficient access to data is inevitable part of effective electricity market [4]. Introduction of new services, appearance of new market players and competition relies on the data availability. Electricity market data are classified into one of three categories: meter data, grid data and market data.

\section{1) Meter Data}

This data refers to all consumers and producers, regardless of the grid level where customer is connected. Meter data is available to all market players at both retail and wholesale market.

\section{2) Grid Data}

It incorporates all data related to transmission and distribution grid operation and maintenance, important for market transactions. Collecting and validation of meter data is 
responsibility of DSO [4]. Free and unlimited data access can represent an important incentive for new market entrants.

\section{3) Market Data}

This class of data includes all types of exogenous data, such as market results, weather data and information about specific assets at customer premises necessary for specific services [4]. Market data also include data types such as unitlevel production/consumption plan for significant grid users, flexibility data (data necessary for the coordination of distributed flexibility resources in a marketplace) and generation data.

\section{Customer Data}

Customer data refers to data produced by a customer and are collected at the customer premises, usually through smart metering system. This class incorporate user and control data, consumption data and production data. Customers that participate at the electricity market, on its own or through aggregator, will make financial benefits and support TSO operation by participation in ancillary services (e.g. flexibility). They will be able to participate at electricity market only if they have access to own smart meter data and if they can authorize third parties to access their data.

\section{IDENTIFICATION OF EXCHANGED DATA WITH USE-CASE METHODOLOGY}

In the scope of TDX-ASSIST project, use-cases have been identified in order to address several technical and business issues based on the state of art on the TSO-DSO interactions in order to be deployed within a suitable ICT infrastructure that supports the collaboration between the stakeholders in the electricity sector $[14,15]$. The use-case development has been done at different abstraction levels, in order to highlight the market issues and DSO interactions.

Business Use Cases (BUCs) are described using technology-agnostic terminology that is invoked by business actors (people or organisations), often working in collaboration, in order to achieve a specific goal that provides clearly defined business value to the actors [14]. In this case, BUCs represent scenarios for the study of improving interoperability, security and scalability between TSOs and DSOs as well as between DSOs and other market participants. It is important to note that BUCs may address different periods associated with regard to different power system operation and management activities, from real-time to yearsahead. They can target issues related to power system operation, operational planning and long-term planning activities simultaneously, especially when inter-temporal dependencies can exist.

While BUCs describe stakeholder interactions at business level with high abstraction, System Use Cases (SUCs) define functional requirements as well as requirements on information models and communication protocols [15]. In the scope of TDX-ASSIST project, 10 Business Use Cases and 18 System Use Cases, using 13 Business Roles, and 20 System Roles are determined (Fig. 3). The BUC and SUC have been defined according to IEC 62559 series and IEC 62913-1 Generic Smart Grid requirements methodology. Some of these
Business and System Roles have been already defined by the Harmonized Electricity Market Role Model, or IEC 61968-1 Interface Reference Model [16].

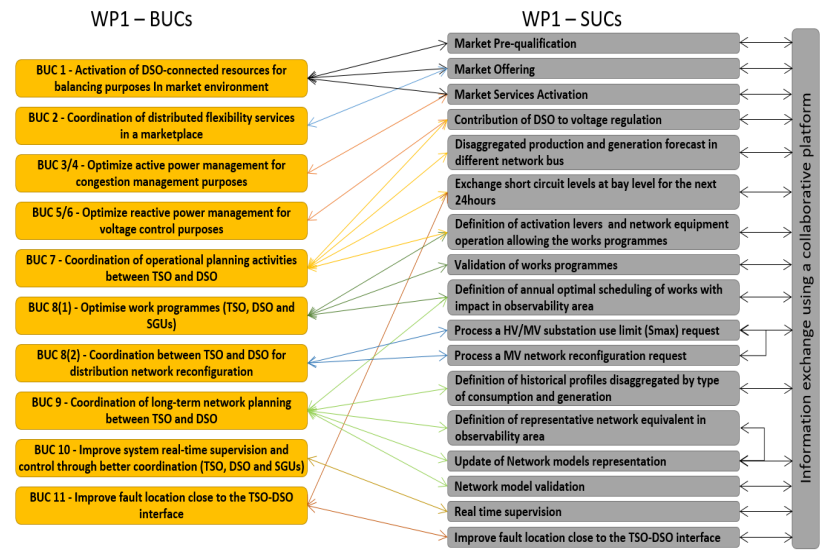

Figure 3. List of Business Use Cases and associated System Use Cases [16]

To ensure interoperability through various processes, two important steps are required: to have a common role model and a common ontology to build a data model. The common ontology to build a data model is based on Use Case description. The standard assessment has been focused on the Business Objects defined in the System Use Cases because they are used for the definition of requirements on the information exchange platform between TSO and DSO [17].

Each SUC proposes a list of information that will be exchanged between TSOs, DSOs and other market participants. Fig. 4 illustrates the standard assessment of BOs, which are further modeled with Common Information Model (CIM) profiles for data exchange between TSO and DSO.

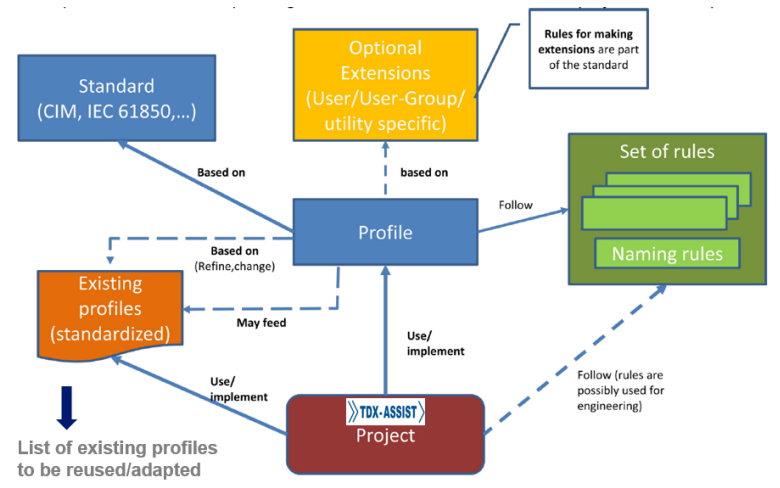

Figure 4. Methodology for TSO-DSO exchanged data modeling using CIM [15]

\section{INTEROPERABLE COMMUNICATION ARCHITECTURE FOR TSO-DSO DATA EXCHANGED: DESIGN PRINCIPLES}

Under new operational and planning conditions, it is likely that TSOs and DSOs will exchange larger amounts of data, with high quality and minimal delays [3]. For this purpose, existing data exchange principles and practices should be reviewed, and new proposed. In this regard, the regulatory framework in Europe has been evolving in the recent past, namely with the release of the Electricity Network Codes (e.g. 
Regulation and Guidelines) and the EU 'Clean Energy for All Europeans' proposal which already set up a common set of requirements for enhancing TSO-DSO data exchange [18].

It is essential that the TSO-DSO data management and exchange was based on an integrated electricity system approach. In that sense, it is important to consider the following issues when designing the information to be exchanged:

- $\quad$ The purpose for the data exchange (e.g. active system management, work programs);

- The time frame in which the data exchange occurs between TSOs and DSOs (real-time operation, operational planning or long-term planning);

- The nature of information exchange (real time, schedule data, structural data);

- The type of information exchanged (states and measures);

- Frequency and granularity of the data to be exchanged;

- IT architecture that supports the information exchange;

- The maximization of usage of available standards to guarantee interoperability in the interface and harmonization of data exchange.

Current ICT architectures and data models for TSO-DSO data exchange are overviewed in [3, 18]. Communication architecture and protocols are developed considering the requirements for the data exchange between TSO and DSO, concerning types of data (real-time data, schedule data and structure data), frequency of data exchange and granularity of data exchange. For each type of data to be exchanged, and depending on data exchange purposes, the frequency of TSODSO data exchange may differ. While for system operation purposes, in which real-time data exchange is of key importance, higher sampling rates of data exchange maybe needed, for operational planning and long-term network planning related activities the frequency under which TSOs and DSOs exchange data maybe much lower.

Communication architectures and concepts, that will be proposed and demonstrated in the scope of TDX-ASSIST project, should enable interoperability with the existing technologies but also leverage advantages of new solutions, such as Internet of Things (IoT). IoT architectures utilize "publish/subscribe" messaging to topics and "brokers" as intermediate switches, which in comparison with widely adopted "client/server" concept introduces better scalability, security and interoperability in data exchange. Application of light IoT protocols, such as MQTT (Message Queue Telemetry Transport) allows data collection from devices with limited processing and storage capabilities over low capacity and unreliable links. MQTT is low-overhead, content agnostic, simple to implement protocol with elementary quality of service (QoS) mechanism. In order to enable secured transport, MQTT relies on security mechanisms enabled at the transport layer, such as TLS (Transport Layer Security). On the other hand, AMQP (Advanced Message Queuing Protocol) is aimed for secure real-time data transmission and business transactions. AMQP, however, suffers from high overhead for real-time communication and implementation at capability constrained devices [19].

Figure 5 presents ICT architecture that provides TSO-DSO exchange of all types data. The presented interoperable communication architecture can be a basis for the demonstration site development in Slovenia, to support implementation of BUC "Activation of DSO-connected resources for balancing purposes in market environment" [14, 15]. In particular, real-time data is exchanged between TSO and DSO by inherited ICCP protocol while other type data are handled through ENTSO-E Communication and Connectivity Service Platform (ECCo SP), which benefits AMQP. ECCo SP platform provides reliable and secured messaging and a file transfer. ECCo SP architecture is consisted of two entities, Energy Communication Platform (ECP) and EDX [3]. ECP provides robust messaging infrastructure founded on AMQP and integrates secure large file exchange through FSSF (File System Shared Folders). EDX is distributed messaging system, built on ECP network. It introduces a concept of services, service providers and consumers. Secured transfer of large file is supported using encryption and HTTP (Hyper Text Transfer Protocol) streaming. In the scope of TDXASSIST project demonstrations, ECCo SP platform will be examined for use case in which balancing service providers (BSP) is placing a bid at the balancing market. TSO (or market platform) sends activation signals to the balancing asset in the distribution grid utilizing the connectivity with BSP through ECCo SP platform. Sharing the same platform, DSO can be aware of activation requests and be able to validate these requests, in the sense that activation shouldn't cause any violation at the current state of the distribution grid. Real-time data related to the activation signals, measurements necessary for activation status monitoring and baseline calculations are sent utilizing MQTT protocol and ECCo SP platform in this scenario. IoT concept is used to collect data metered in the distribution grid (such as smart meters and power quality meters) and make this data available to variety of business and technical applications through the "broker". MQTT imposes itself as efficient communication protocol for real-time data collection from PQ meters and smart meters located in the distribution grid. Historical measurements on the consumption and voltage in the distribution grid can be further employed by demand response service provision by distribution network operator (DNO), such as conservation voltage reduction (CVR). Common Information Model (CIM) semantic is used to exchange data, what enables interoperability and scalability among heterogeneous services and devices existing in smart grids. Cyber Security standards, such as parts of IEC 6235, must be implemented. ECCo SP platform leverages IEC standards like IEC 62325-503 (MADES), that it will be compliant soon with IEC standard 61968-100.

Performance of ECCoSp platform is preliminary examined and results are given in Fig. 6. Latency is measured between central ECCo SP side and three different client sides. Even though performance is impacted by the quality of 
communication links and used servers, it can be observed that variance between clients is present when a large number of small files is exchanged.

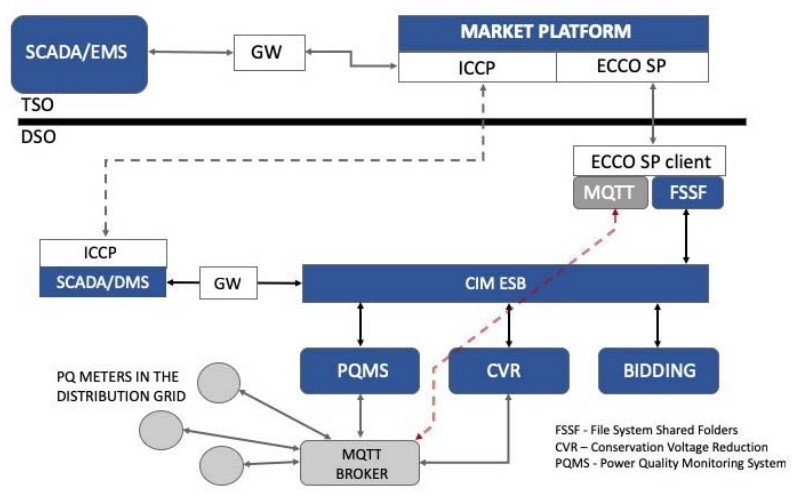

Figure 5. ICT architecture to support demand response meachanism in market enviroment (Example for Slovenian demonstrator)

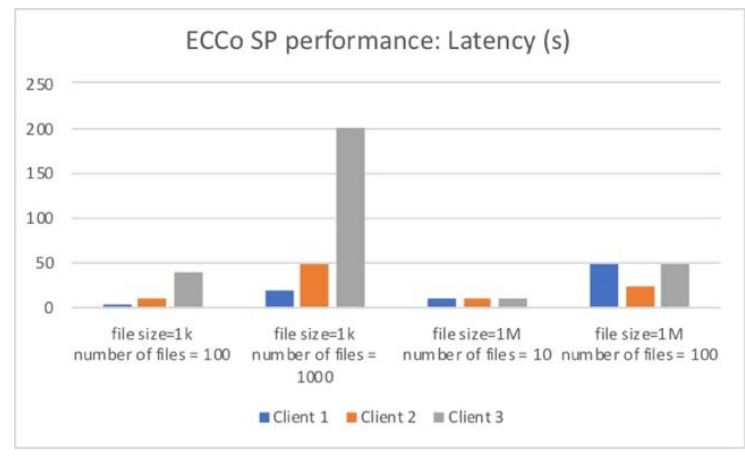

Figure 6. Measured latency of ECCo SP platform

\section{CONCLUSION}

This paper describes a model-driven methodology in order to introduce an interoperable communication architecture supporting TSO-DSO information exchange. The methodology is based on a set of international standards such as: IEC Use Case methodology, Smart Grid Reference Architecture which leverages previous work conducted at the European level through M/490 Smart Grid Mandate (Sustainable Process working groups, Reference Architecture working group). A set of Business Objects are determined after definition of Business and System Use Cases. The Business Objects associated to SUCs are analyzed against existing CIM Profiles. If there is no existing profile supporting some Business Objects, new CIM profiles can be derived, and some CIM extensions can be proposed to IEC standard organizations. These profiles will then be implemented using a Secure Communication Infrastructure, founded on new technologies as well as already available platforms like ECCo SP, provided by ENTSO-E.

\section{ACKNOWLEDGMENT}

This work is part of the TDX-ASSIST project (www.tdxassist.eu) activities. This project has received funding from the
European Union's Horizon 2020 research and innovation programme under grant agreement No 774500.

\section{REFERENCES}

[1] M. Richter, "Utilities' business models for renewable energy: A review", Renewable and Sustainable Energy Reviews, vol. 16, pp. 2483-2493, June 2012.

[2] M. Zipf and D. Most, "Cooperation of TSO and DSO to provide ancillary services", in Proc. 2016 International Conference on the European Energy Market (EEM), pp. 1-6.

[3] E. Lambert, et al., "State of the Art - TSO-DSO Interoperability," Deliverable D1.1, H2020 TDX-ASSIST, Dec. 2017.

[4] THEMA Consulting Group (2017, June). Data Exchange in electric power systems: European State of Play and Perspective, 2017, [Online]. Available:

https://www.entsoe.eu/Documents/News/THEMA Report 201703 web.pdf

[5] EDSO, Eurelectric, GEODE, CEDEC, and ENTSO-E (2016, July). "TSO - DSO Data Management Report". [Online]. Available: https://www.entsoe.eu/Documents/Publications/Position\%20papers\%20 and\%20reports/entsoe TSO-DSO DMR web.pdf

[6] H. Gerard, E. I. Rivero Puente and D. Six, "Coordination between transmission and distribution system operators in the electricity sector: A conceptual framework", Utilities Policy, vol. 50, pp. 40-48, Feb. 2018.

[7] Smart Grid Reference Architecture, CEN-CENELEC-ETSI, Nov. 2012.

[8] ENTSO-E (2015). Towards smarter grids: Developing TSO and DSO roles and interactions for the benefit of consumers. [Online]. Available: https://www.entsoe.eu/Documents/Publications/Position\%20papers\%20 and\%20reports/150303 ENTSO-E Position Paper TSODSO interaction.pdf

[9] EDSO (2015, May). Coordination of transmission and distribution system operators: a key step for the Energy Union. [Online]. Available: https://www.edsoforsmartgrids.eu/wpcontent/uploads/public/Coordination-of-transmission-and-distributionsystem-operators-May-2015.pdf

[10] EDSO (2015, May). General Guidelines for Reinforcing the Cooperation Between TSOs and DSOs. [Online]. Available: https://www.entsoe.eu/Documents/Publications/Position\%20papers\%20 and\%20reports/entsoe pp TSO-DSO web.pdf

[11] European Commission (2017, June). EU energy in figures - Statistical Pocketbook 2017. [Online]. Available: https://ec.europa.eu/energy/sites/ener/files/documents/pocketbook_ener gy 2017 web.pdf

[12] Electric Power Research Institute (2014). The Integrated Grid Realizing the Full Value of Central and Distributed Energy Resources. [Online]. Available: https://www.eenews.net/assets/2014/02/10/document cw 02.pdf

[13] N. Suljanovic, et al., "Survey report - Evolving data access needs," Deliverable D3.1, H2020 TDX-ASSIST, Mar. 2018.

[14] H. Morais, et al., "TSO-DSO Use Cases," Deliverable D1.2, H2020 TDX-ASSIST, Mar. 2018.

[15] J. Cantenot, et al., "TSO-DSO Use Cases," Deliverable D1.3, H2020 TDX-ASSIST, Sep. 2018.

[16] ENTSO-E, Harmonised Electricity Role Model, [Online]. Available: https://www.entsoe.eu/digital/cim/role-models/

[17] H. Morais et al, "Balancing Services Business Use Case Development for TSO-DSO Interoperability Demonstration", in Proc. 2018 Mediterian Conference on Power Generation, Transmission, Distribution and Energy Conversion (MEDPOWER), pp. 1-5.

[18] E. Lambert et al, "Practices and architectures for TSO-DSO data exchange: European landscape", in Proc. 2018 IEEE PES Innovative Smart Grid Technologies Europe (ISGT), pp. 1-6.

[19] N. Naik, "Choice of Effective Messaging Protocols for IoT Systems: MQTT, CoAP, AMQP and HTTP", in Proc. 8th IEEE International Systems Engineering Symposium (ISSE), pp. 1-7, 2018 